\title{
Direct Polymerase Chain Reaction Detection of Campylobacter jejuni and Campylobacter coli in Raw Milk and Dairy Products
}

B. WEGMÜLLER, J. LÜTHY, AND U. CANDRIAN

Laboratory of Food Chemistry, Institute of Biochemistry, University of Bern, Freiestrasse 3, 3012 Bern, Switzerland

Volume 59 , no. 7 , p. 2161 to 2165 : In contrast to the information given in the paper, only the 54 raw milk samples were analyzed by the culture method. In addition, the composition of the culture media was not correctly reported. The selective supplement used for the Campylobacter enrichment broth was not according to Preston, but the Skirrow Selective Supplement (Oxoid, Basingstoke, England) was used. Campylobacter selective agar was based on Blood Agar Base (Oxoid) supplemented with Butzler Selective Supplement (Oxoid) instead of Preston Selective Supplement.

\section{Degradation and Induction Specificity in Actinomycetes That Degrade $p$-Nitrophenol}

L. F. HANNE, L. L. KIRK, S. M. APPEL, A. D. NARAYAN, AND K. K. BAINS

Departments of Biology and Chemistry, California State University, Chico, California 95929-0515

Volume 59, no. 10, p. 3506, column 1: Figure 1 should appear as shown below.

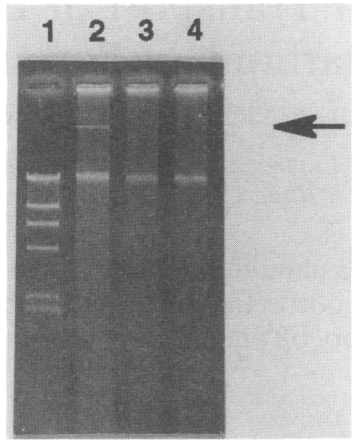

FIG. 1. Agarose gel electrophoresis of plasmid DNA isolated from wild-type and cured $A$. aurescens. Lanes: $1, \lambda$ HindIII markers; $2, A$. aurescens TW17; $3, A$. aurescens TW17C1; 4, $A$. aurescens TW17C2. The arrow indicates the plasmid band which correlates with the PNP degradation phenotype.

\section{New Medium for the Simultaneous Detection of Total Coliforms and Escherichia coli in Water \\ KRISTEN P. BRENNER, CLIFFORD C. RANKIN, YVETTE R. ROYBAL, GERARD N. STELMA, JR., PASQUALE V. SCARPINO, AND ALFRED P. DUFOUR \\ Environmental Monitoring Systems Laboratory, U. S. Environmental Protection Agency, Cincinnati, Ohio 45268, and Department of Civil and Environmental Engineering, University of Cincinnati, Cincinnati, Ohio 45221}

Volume 59, no. 11, p. 3534, last sentence of the abstract. This sentence should read as follows. “This selective and specific medium, which employs familiar membrane filter technology to analyze several types of water samples, is less expensive than the liquid chromogen and fluorogen media and may be useful for compliance monitoring of drinking water." 